\title{
A Mathematical Model for Finding Bivariate Normal distribution in normal women for Luteinizing Hormone and Progesterone
}

\author{
Dr.S.Lakshmi * and M.Agalya** \\ *Principal, Govt.Arts and Science College, Peravurani ,Thanjavur Dist. \\ ** Research Scholar,PG\& Research Department of Mathematics, K.N. Govt.Arts College for \\ Women,Thanjavur.
}

\begin{abstract}
A very important property of jointly random normal variables and which will be starting point for our development, is done zero correlation implies independent . This property can be verified by using multivariate transform .suppose $\mathrm{U}$ and $\mathrm{V}$ are independent zero mean normal random variables and that $\mathrm{X}=$ $\mathrm{aU}+\mathrm{bV}$ and $\mathrm{Y}=\mathrm{cU}+\mathrm{dV}$, so that $\mathrm{X}$ and $\mathrm{Y}$ are jointly normal. The exponent term $\mathrm{q}(\mathrm{x}, \mathrm{y})$ is the quadratic form of $\mathrm{x}$ and $\mathrm{y}$ is obtained.The more general case when $\mathrm{x}$ and $\mathrm{y}$ are dependent a typical contour is described by an ellipse and it is utilized for our application part.

The original definition of LPD is a corpus luteum defective in progesterone secretion,which in turn was a cause of infertility or early spontaneous abortion. Here we have considered Luteinizing hormone (LH) and Progesterone as Bivariate normal and corresponding quadratic function $\mathrm{q}(\mathrm{x}, \mathrm{y})$ is obtained when they are correlated.
\end{abstract}

Keywords: - LH, FSH, Bi- Variate Normal Distribution Mathematical Classification: $60 G_{X X}, 60 E 05$

\section{THE MATHEMATICAL MODEL}

Degradation model for the secretion of Progesterone in luteal Phase deficiency women, is developed by Lakshmi.S and Agalya.M [5].

Let $\mathrm{U}$ and $\mathrm{V}$ be two random variables, and consider two new random variables $\mathrm{X}$ and $\mathrm{Y}$ of the form

$\mathrm{X}=\mathrm{a} \mathrm{U}+\mathrm{b} \mathrm{V}$

$\mathrm{Y}=\mathrm{c} \mathrm{U}+\mathrm{d} \mathrm{V}$,

Where a,b,c,d are some scalars.Each one of the random variables $\mathrm{X}$ and $\mathrm{Y}$ is normal, since it is a linear function of independent normal random variables. Furthermore, because $\mathrm{X}$ and $\mathrm{Y}$ are linear functions of the same two independent normal variables, their joint PDF takes a special form, known as the bi- variate normal PDF. The bi- variate normal PDF has several useful and elegant properties and, for this reason, it is a commonly employed model. In this section we derive many such properties, both qualitative and analytical, culminating in a closed- form expression for the joint PDF.

\subsection{The Conditional Distribution of $\mathrm{X}$ Given $\mathrm{Y}$}

We now turn to the problem of estimating $\mathrm{X}$ given the value of $\mathrm{Y}$. We assume that both $\mathrm{X}$ and $\mathrm{Y}$ have positive variance. Let us define

$$
\begin{array}{ccc}
\hat{X}=\rho \frac{\sigma_{X}}{\sigma_{Y}} \mathrm{Y}, & \tilde{X}=X-\hat{X}, \\
\text { where } & \rho=\frac{E[X Y]}{\sigma_{X} \sigma_{Y}}
\end{array}
$$

is the correlation coefficient of $\mathrm{X}$ and $\mathrm{Y}$. Since $\mathrm{X}$ and $\mathrm{Y}$ are linear combinations of independent normal random variables $\mathrm{U}$ and $\mathrm{V}$, it follows that $\mathrm{Y}$ and $\tilde{X}$ are also linear combinations of $\mathrm{U}$ and $\mathrm{V}$. In Particular, $\mathrm{Y}$ and $\tilde{X}$ are jointly normal.

Furthermore,

$$
\mathrm{E}[Y \tilde{X}]=\mathrm{E}[Y X]-\mathrm{E}[Y \hat{X}]=\rho \sigma_{X} \sigma_{Y}-\rho \frac{\sigma_{X}}{\sigma_{Y}} \sigma_{Y}^{2}=0
$$

Thus $\mathrm{Y}$ and $\tilde{X}$ are uncorrelated and, therefore, independent. Since $\hat{X}$ is a scalar multiple of $\mathrm{Y}$, it follows that $\hat{X}$ and $\widetilde{X}$ are independent.

We have so far decomposed $X$ into a sum of two independent normal random variables, namely,

$$
\mathrm{X}=\hat{X}+\tilde{X}=\rho \frac{\sigma_{X}}{\sigma_{Y}} Y+\tilde{X} .
$$

We take conditional expectations of both sides, given $\mathrm{Y}$, to obtain

$$
\mathrm{E}[X \mid Y]=\rho \frac{\sigma_{X}}{\sigma_{Y}} E[Y \mid Y]+E[\widetilde{X} \mid Y]=\rho \frac{\sigma_{X}}{\sigma_{Y}} Y=\hat{X},
$$


where we have made use of the independence of $\mathrm{Y}$ and $\tilde{X}$ to set $\mathrm{E}[\tilde{X} \mid Y]=0$. We have therefore reached the important conclusion that the conditional expectation $\mathrm{E}[X \mid Y]$ is a linear function of the random variable $\mathrm{Y}$.

Using the above decomposition, it is now easy to determine the conditional PDF of X. Given a value of $Y$, the random variable $\hat{X}=\rho \sigma_{X} Y / \sigma_{Y}$ becomes a known constant, but the normal distribution of the random variable $\tilde{X}$ is unaffected, since $\tilde{X}$ is independent of $\mathrm{Y}$ [4].Therefore, the conditional distribution of $\mathrm{X}$ given $\mathrm{Y}$ is the same as the unconditional distribution of $\tilde{X}$, shifted by $\hat{X}$. Since $\tilde{X}$ is normal with mean zero and some variance $\sigma_{\tilde{X}}^{2}$, we conclude that the conditional distribution of $\mathrm{X}$ is also normal with mean $\hat{X}$ and the same variance $\sigma_{\widetilde{X}}^{2}[11,12]$. The variance of $\tilde{X}$ can be found with the following calculations:

$$
\begin{aligned}
& \quad \sigma_{X}^{2}=E\left[\left(X-\rho \frac{\sigma_{X}}{\sigma_{Y}} Y\right)^{2}\right] \\
& =\sigma_{X}^{2}-2 \rho \frac{\sigma_{X}}{\sigma_{Y}} \rho \sigma_{X} \sigma_{Y}+\rho^{2} \frac{\sigma_{X}^{2}}{\sigma_{Y}^{2}} \sigma_{Y}^{2} \\
& =\left(1-\rho^{2}\right) \sigma_{X}^{2},
\end{aligned}
$$

where we have made use of the property $E[X Y]=\rho \sigma_{X} \sigma_{Y}$.

\section{The Form of the Bivariate Normal PDF:}

Having determined the parameters of the PDF of $\tilde{X}$ and of the conditional PDF of $\tilde{X}$, we can give explicit formulas for these PDFs. We keep assuming that $\mathrm{X}$ and $\mathrm{Y}$ have zero means and positive variances. Furthermore, to avoid the degenerate where $\tilde{X}$ is identically zero, we assume that $|\rho|<1 \quad[9,10]$.We have

$$
f_{X}(\tilde{x})=f_{\widetilde{X} \mid Y}(\tilde{x} \mid y)=\frac{1}{\sqrt{2 \pi} \sqrt{1-\rho^{2}} \sigma_{X}} e^{-\tilde{x}^{2} / 2 \sigma_{\tilde{X}}^{2}}
$$

and

$f_{X \mid Y}(x \mid y)=\frac{1}{\sqrt{2 \pi} \sqrt{1-\rho^{2}} \sigma_{X}} e^{-\left(x-\rho \frac{\sigma_{X}}{\sigma_{Y}} y\right)^{2}} / 2 \sigma_{X}^{2}$,

where

$$
\sigma_{\tilde{X}}^{2}=\left(1-\rho^{2}\right) \sigma_{X}^{2}
$$

Using also the formula for the PDF of $\mathrm{Y}$,

$$
f_{Y}(y)=\frac{1}{\sqrt{2 \pi} \sigma_{Y}} e^{-y^{2} / 2 \sigma_{Y}^{2}}
$$

and the multiplication rule $f_{X, Y}(x, y)=f_{Y}(y) f_{X \mid Y}(x \mid y)$, we can obtain the joint $\quad$ PDF of X and Y. This PDF is of the form

where the normalizing constant is

$$
f_{X, Y}(x, y)=c e^{-q(x, y)},
$$

$$
c=\frac{1}{2 \pi \sqrt{1-\rho^{2}} \sigma_{X} \sigma_{Y}} .
$$

The exponent term $\mathrm{q}(\mathrm{x}, \mathrm{y})$ is a quadratic function of $\mathrm{x}$ and $\mathrm{y}$,

$$
q(x, y)=\frac{y^{2}}{2 \sigma_{Y}^{2}}+\frac{\left(x-\rho \frac{\sigma_{X}}{\sigma_{Y}} y\right)^{2}}{2\left(1-\rho^{2}\right) \sigma_{X}^{2}}
$$

which after some straightforward algebra simplifies to

$$
q(x, y)=\frac{\frac{x^{2}}{\sigma_{X}^{2}}-2 \rho \frac{x y}{\sigma_{X} \sigma_{Y}}+\frac{y^{2}}{\sigma_{Y}^{2}}}{2\left(1-\rho^{2}\right)} .
$$

An important observation here is that the joint PDF is completely determined by $\sigma_{X}, \sigma_{Y}$ and $\rho$.

In the special case where $\mathrm{X}$ and $\mathrm{Y}$ are uncorrelated $(\rho=0)$, this joint PDF takes the simple form

$$
f_{X, Y}(x, y)=\frac{1}{2 \pi \sigma_{X} y} e^{\frac{-x^{2}}{2 \sigma_{X}^{2}}}-\frac{y^{2}}{2 \sigma_{Y}^{2}},
$$

Which is just the product of two independent normal PDFs. We can get some insight into the form of this PDF by considering its contours, i.e.,sets of points at which the PDF takes a constant value. These contours are described by an equation of the form

$$
\frac{x^{2}}{\sigma_{X}^{2}}+\frac{y^{2}}{\sigma_{Y}^{2}}=\text { constant }
$$

and are ellipses whose two axes are horizontal and vertical.

In the more general case where $\mathrm{X}$ and $\mathrm{Y}$ are dependent, a typical contour is described by

$$
\frac{x^{2}}{\sigma_{X}^{2}}-2 \rho \frac{x y}{\sigma_{X} \sigma_{Y}}+\frac{y^{2}}{\sigma_{Y}^{2}}=\text { constant }
$$

and is again an ellipse, but its axes are no longer horizontal and vertical.

\section{APPLICATION}


In an era of ever- increasing knowledge of the symphony of physiologic events surrounding ovulation, fertilization and implantation of human oocytes and embryos, born out of previously unimaginable technologic advancement, few scientific puzzles have been explored more times than the entity synonymously known as luteal phase insufficiency, inadequacy, defect, or deficiency. This paper uses the term luteal phase deficiency (LPD) .

Definition of LPD: The original definition of LPD is a corpus luteum defective in progesterone secretion, which in turn was a cause of infertility or early spontaneous abortion. Further investigation led to a broadening of this definition to include a short luteal phase interval ( $<12$ days between ovulation and menses) with relatively normal progesterone concentration, a normal-length luteal phase with inadequate progesterone production, or inadequate endometrial response to otherwise normal progesterone concentration [7]. Because of the possibility sporadic abnormalities of luteal function in otherwise fertile women, most experts agree that the diagnosis of LPD can be made only after repeated testing. LPD is relevant in the clinical setting only if it is present in most menstrual cycles in a patient. The requirement for two consecutive menstrual cycles, as currently applied to the diagnosis of LPD, is arbitrary .

\subsection{Prevalence and Incidence:}

An accurate estimation of the true prevalence of a disease requires an understanding of the sensitivity of diagnostic modalities and a definition of the abnormality. In case of LPD, in which the diagnosis has been based variably on single or multiple serum progesterone measurements, urinary progesterone metabolites, salivary progesterone levels and endometrial histology, the true prevalence is in dispute. Estimating the incidence of LPD in a normal, fertile population is extremely difficult because of the apparently sporadic nature of the disorder. Much of the difficulty centers around the circular pattern of the verification of one test by comparison with another unproven test with inherent and immeasurable bias. Also, there is lack of uniformity of the standards for specific test used for diagnosis (e.g., endometrial biopsy specimen out- of- phase by $\geq 2$ days verses $\geq 3$ days ).

Despite these obstacles, several reports in the medical literature have attempted to provide estimates of prevalence. In a review of clinical experience, reported LPD diagnosed by out- of- phase ( $\geq 3$ days ) endometrial biopsy specimen in $3.5 \%$ of infertile patients and $35 \%$ of cases recurrent miscarriage. Using similar methods we have found that $13.5 \%$ of infertile patients had evidence of histologically diagnosed LPD, with an incidence of $32.5 \%$ for recurrent miscarriage. The later results were supported by findings of other studies, $[1,5]$ despite failing to account for differences in the definition of an out-of- phase biopsy specimen.

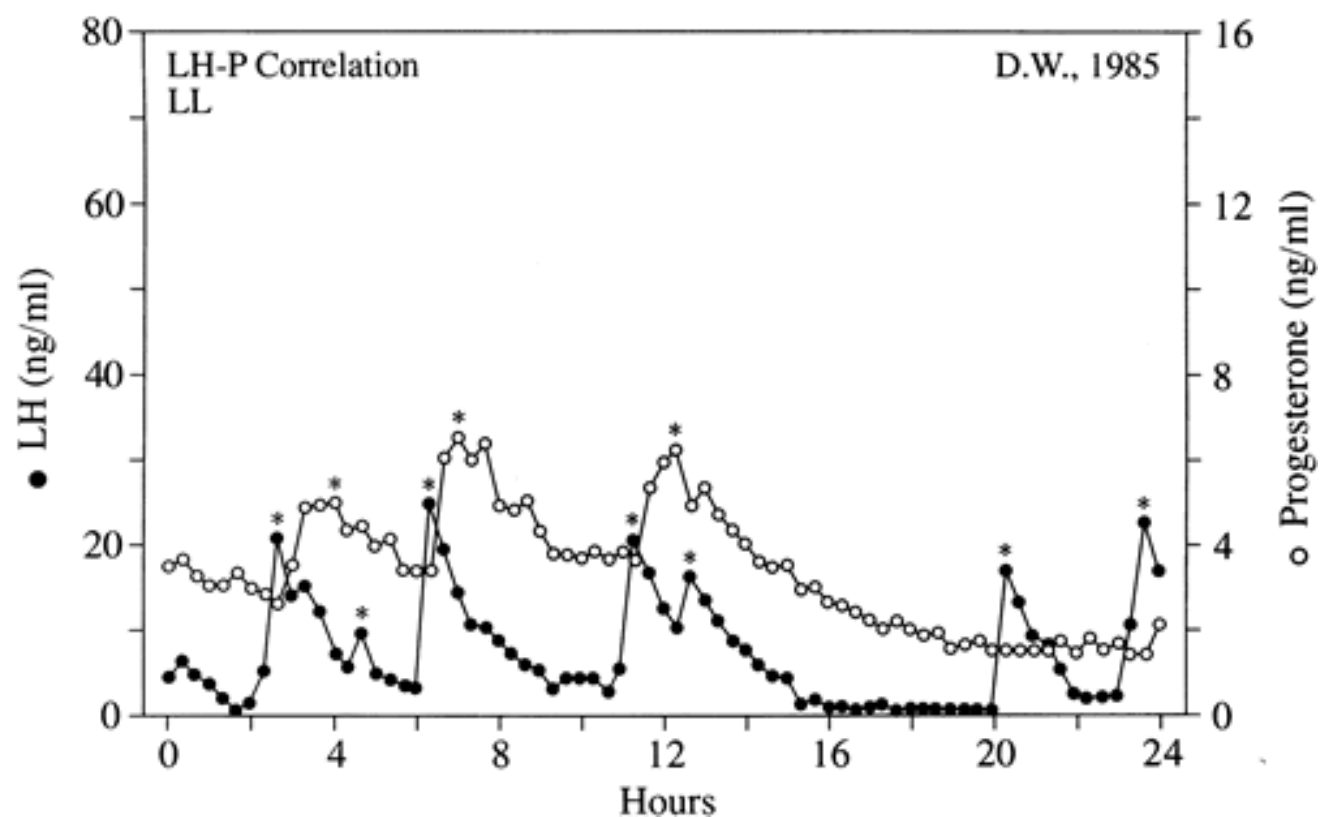

Fig 1: Simultaneous luteinizing hormone and progesterone secretion patterns in the late luteal cycle phase in a normal woman .Secretory events that were identified as pulses are indicated by asterisks for luteinizing hormone and progesterone. In the mid to late luteal phases of the cycle, there is synchrony between luteinizing hormone and progesterone secretion. 
Normal corpus luteum formation and function begin in the follicular phase with recruitment of a cohort of growing follicles primarily under the influence of follicle- stimulating hormone (FSH).

Two distinct steroidogenic cell types have been identified with the human corpus luteum: large luteal cells derived from the granulosa cells (granulosa lutein cells), which produce large quantities of progesterone but are not LH receptive and account for basal progesterone secretion, and small luteal cells derived from thecal cells ( theca lutein cells), which are LH receptive in the second half of the luteal phase and are responsible for pulsatile changes in circulating progesterone levels Fig 1 [2,3].During the first half of the luteal phase, increased amounts of estradiol and progesterone are secreted by the corpus luteum with no temporal correlation to LH pulses, primarily by the large granulosa lutein cells. During the second half of the luteal phase, estradiol and progesterone levels in the blood fluctuate in phase with LH pulses, presumably as a result of intermittent secretion by the small lutein cell Population [8].

\section{THE MATHEMATICAL RESULTS}

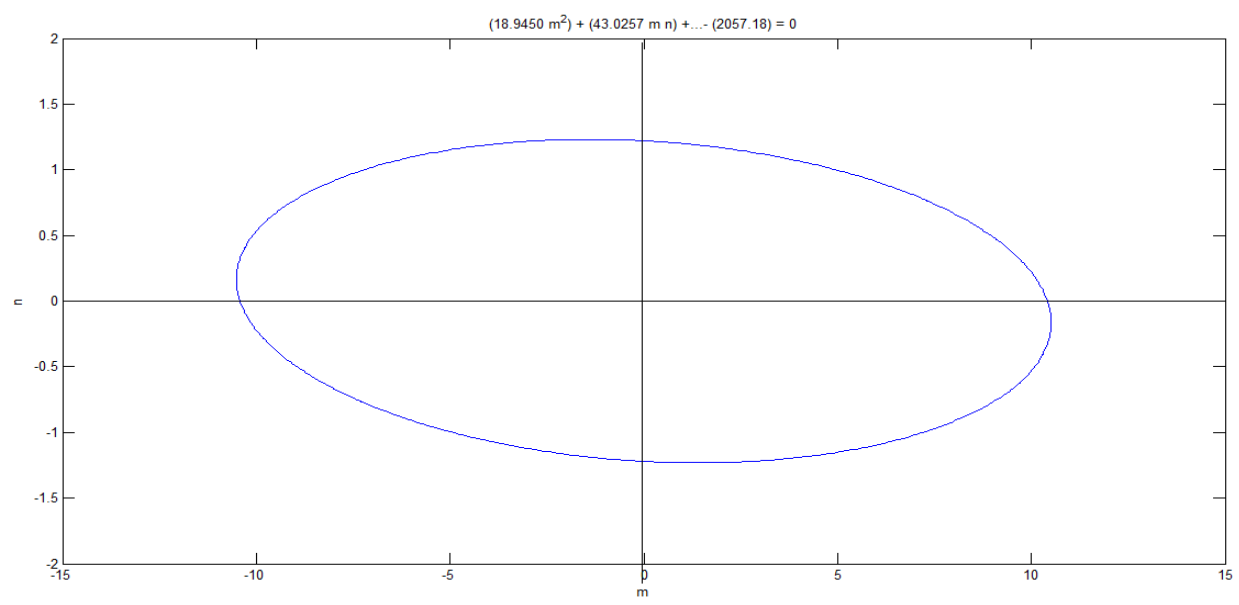

Fig 3.1: A contour of the Bi-variate normal PDF of the LH corresponds to normal women in case of negative correlation coefficient $\rho$.

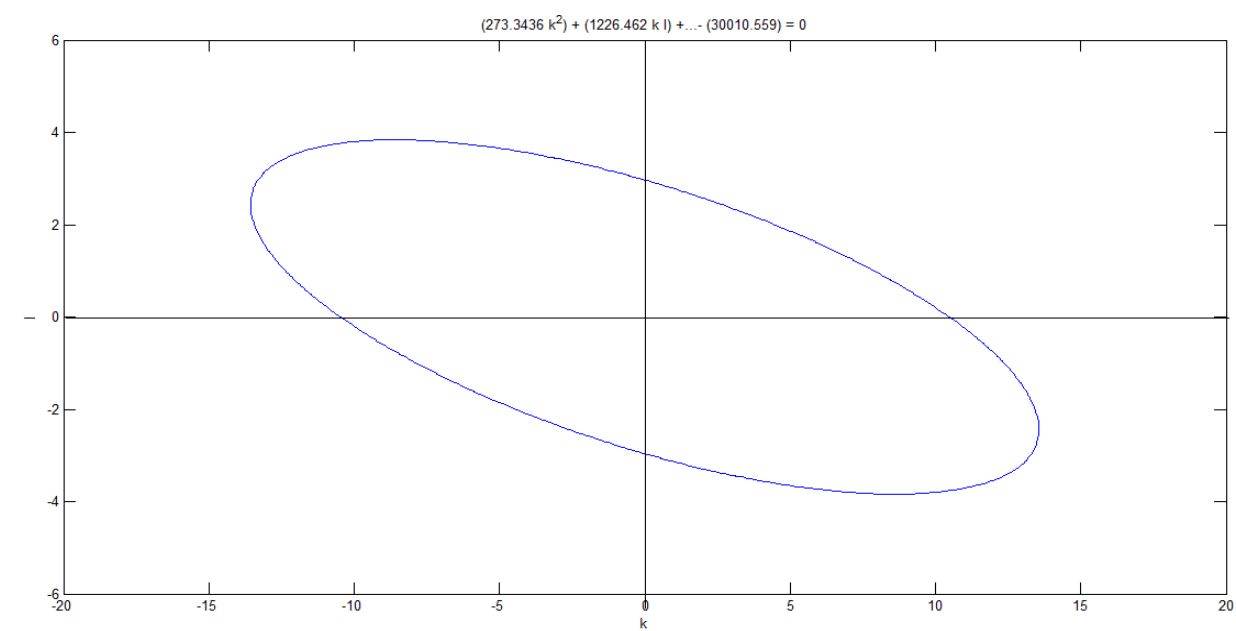

Fig 3.2: A contour of the Bi-variate normal PDF of the Progesterone corresponds to normal women in case of negative correlation coefficient $\rho$.

\section{CONCLUSION}

If $\mathrm{X}$ and $\mathrm{Y}$ are jointly normal, then each random variable $\mathrm{X}$ and $\mathrm{Y}$ is normal. If $\mathrm{X}$ and $\mathrm{Y}$ were jointly normal, we have a contradiction to our conclusion that zero correlation implies independence. It follows that $\mathrm{X}$ and $\mathrm{Y}$ are not jointly normal, even though both marginal distribution are normal. A contour of the Bi-variate normal PDF of LH and Progesterone corresponds to a negative correlation coefficient $\rho$. These contours are described by an ellipse, but its axes are horizontal and vertical. 


\section{REFERENCES}

[1] Hinney B, Henze C, Kuhn W, Wuttke W: The corpus luteum insufficiency: A multifactorial disease. J Clin Endocrinol Metabol 81:565, 1996.

[2]. Hsu CC, Kuo HC, Wang ST, Huang KE: Interference with uterine blood flow by clomiphene citrate in women with unexplained infertility. Obstet Gynecol 86:917, 1995.

[3]. Jobanputra K, Toner JP, Denoncourt R, Gibbons WE: Crinone 8\% (90 mg) given once daily for progesterone replacement therapy in donor egg cycles. Fertil Steril 72:980, 1999.

[4]. Kotz, S.; Balakrishnan, N.; and Johnson, N.L.”Bivariate and Trivariate Normal Distributions".Ch 46 in Continuous Multivariate Distribution,Vol.1:Models and Aplications, $2^{\text {nd }}$ ed.New York: wiley,PP.251348,2000 .

[5]. Lakshmi,S. and Agalya,M.'The Mathematical Model on Degradation of Progesterone in Luteal Phase Deficiency Women".JJAMMS, Vol.2, No.1 (Jan-June 2013),pp.1-9.

[6]. Lessey BA, Yeh I, Castelbaum AJ, et al: Endometrial progesterone receptors and markers of uterine receptivity in the window of implantation. Fertil Steri1 65:477, 1996.

[7]. Li T, Dockery P, Cooke I: Endometrial development in the luteal phase of women with various types of infertility: Comparison with women of normal fertility. Hum Reprod 6:325, 1991.

[8]. Ludwig M, Klein HH, Diedrich K, Ortmann O: Serum leptin concentrations throughout the menstrual cycle. Arch GynecolObstet 263:99, 2000.

[9]. Rose,C. and smith,M.D." The Bivariate Normal".\$6.4 A in Mathematical Statistics with Mathematica.New York: Springer- Verlag,pp.216-226,2002.

[10]. Rose,C. and smith, M.D. "The Multivariate Normal Distribution". Mathematica J.6,32-37,1996.

[11]. Spiegel,M.R. Theory and Problems of Probability and statisti.New York: Mc Graw-Hill,p.118,1992.

[12]. Stuart, A.; and Ord, J.K.kendall's Advanced Theory of statistics, Vol.1: Distribution Theory $6^{\text {th }}$ ed .New York:Oxford University Press, 1998. 